\title{
ARTICLE MicroRNA-219a-5p suppresses intestinal inflammation through inhibiting Th1/Th17-mediated immune responses in inflammatory bowel disease
}

\author{
Yan Shi ${ }^{1}$, Shenglan Dai ${ }^{1}$, Caiyu Qiu ${ }^{1}$, Tao Wang ${ }^{1}$, Yong Zhou ${ }^{1}$, Cuihua Xue ${ }^{1}$, Jun Yao ${ }^{1}$ and Yaping $\mathrm{Xu}^{1}$
}

\begin{abstract}
MicroRNA (miR)-219a-5p has been implicated in the development of numerous progression of carcinoma and autoimmune diseases. However, whether miR-219a-5p is involved in the pathogenesis of inflammatory bowel disease (IBD) remains elusive. In this study, we demonstrated that miR-219a-5p expression was significantly decreased in the inflamed intestinal mucosa and peripheral blood (PB)-CD4 ${ }^{+}$T cells from patients with IBD. Proinflammatory cytokines (e.g., IL-6, IL-12, IL-23 and TNF-a) inhibited miR-219a-5p expression in CD4 ${ }^{+}$T cells in vitro. Lentivirus-mediated miR-219a-5p downregulation facilitated Th1/Th17 cell differentiation, whereas miR-219a-5p overexpression exerted an opposite effect. Luciferase assays confirmed that ETS variant 5 (ETV5) was a functional target of miR-219a-5p and ETV5 expression was significantly increased in the inflamed intestinal mucosa and PB-CD4 ${ }^{+}$T cells from IBD patients. ETV5 overexpression enhanced Th1/Th17 immune response through upregulating the phosphorylation of STAT3 and STAT4. Importantly, supplementation of miR-219a-5p ameliorated TNBS-induced intestinal mucosal inflammation, characterized by decreased IFN- $\gamma^{+} \mathrm{CD}^{+} \mathrm{T}$ cells and IL-17A ${ }^{+} \mathrm{CD} 4^{+} \mathrm{T}$ cells infiltration in the colonic lamina propria. Our data thus reveal a novel mechanism whereby miR-219a-5p suppresses intestinal inflammation through inhibiting Th1/Th17mediated immune responses. miR-219a-5p might be a target for the treatment of IBD.
\end{abstract}

Mucosal Immunology (2020) 13:303-312; https://doi.org/10.1038/s41385-019-0216-7

\section{INTRODUCTION}

Inflammatory bowel diseases (IBD), including Crohn's disease (CD) and ulcerative colitis (UC), is a group of complex immunemediated chronic and relapsing inflammatory disorders in gastrointestinal tract. ${ }^{1,2}$ Inflammation in $C D$ patients mainly located in the terminal ileum, while inflammation in patients with UC is confined to the colon. Although the exact etiology and pathogenesis of IBD remain unclear, it has been demonstrated that genetic susceptibility, environmental factors, and microbial flora dysbiosis are associated with the dysregulated excessive immune response and intestinal mucosal damage in IBD. $^{3-5}$ This sustained and aberrant immune response is considered to be mainly facilitated by the dysfunction of the intestinal epithelial barrier and defects in the regulation of intestinal mucosal immunity. 6,7

Accumulating evidence demonstrates that infiltration of abnormally activated $\mathrm{CD}^{+} \mathrm{T}$ cells in colon are central features of IBD, and $\mathrm{CD}^{+}{ }^{+}$T-cell-mediated inflammation is thought to play critical roles in the pathogenesis of IBD and experimental colitis. ${ }^{8-10} \mathrm{CD}^{+}$ T cells mainly include T helper cell type 1 (Th1), Th2, Th17 and regulatory $T$ (Treg) cells. Classically, $C D$ was thought to be mediated by interferon (IFN)- $\gamma$-producing Th1 cells, and UC was associated with Th2-cell-mediated immune response. Nevertheless, and more recently, Th17 cells, a new subtype of CD4 ${ }^{+} \mathrm{T}$ cells capable of producing proinflammatory cytokines (e.g., IL-17A, IL21, TNF-a), have been demonstrated to play key roles in the contribution of intestinal inflammation in both CD and UC. ${ }^{11,12}$
MicroRNAs (miRNAs) are a group of highly conserved small noncoding RNAs consisting of about 22 nucleotides that regulate gene expression through binding to the $3^{\prime}$-untranslated (UTR) region of the target gene. ${ }^{9,13} \mathrm{~A}$ single miRNA can simultaneously target various mRNAs, thereby regulating gene expression from the respective genes. To date, there are compelling evidences that various miRNAs are differentially expressed in the peripheral blood mononuclear cells (PBMCs) and intestinal mucosa of IBD and contribute to the development of colitis via regulating the differentiation of immune cells and production of inflammatory mediators. ${ }^{14-20}$ miR-665 has been demonstrated to be significantly upregulated in the inflamed mucosa of IBD patients and colitic mice. Downregulation of miR-665 could inhibit inflammation-induced intestinal epithelial cell apoptosis both in vivo and in vitro. ${ }^{15}$ Recent studies demonstrated that miR-155 is increased in IBD and miR-155 deficiency ameliorates experimental colitis via inhibiting Th1/Th17 responses and regulating macrophage polarization. ${ }^{19,20}$ However, the way in which miRNAs regulate intestinal inflammatory responses in IBD remains largely unknown.

miR-219a-5p has been implicated in the development of numerous progression of carcinoma and autoimmune diseases. A previous study identified that miR-219a-5p is undetectable in cerebrospinal fluid of multiple sclerosis (MS) patients compared with healthy controls, suggesting that miR-219a-5p may be a candidate biomarker for the diagnosis of $M S^{21}$ Recently, miR-219a$5 p$ expression was found to be significantly decreased in spinal neurons from complete Freund's adjuvant (CFA)-induced chronic

'Department of Gastroenterology, Affiliated People's Hospital of Jiangsu University, Zhenjiang 212002, China

Correspondence: Jun Yao (YaojunYRY@163.com) or Yaping Xu (xyp1201@163.com)

Received: 2 April 2019 Revised: 11 September 2019 Accepted: 28 September 2019

Published online: 18 October 2019 
inflammation pain mice model. ${ }^{22}$ Supplementation of exogenous miR-219a-5p significantly reversed chronic inflammatory pain spinal neuronal sensitization, indicating a therapeutic effect of miR-219a$5 p$ on chronic inflammatory pain. miR-219a-5p contributes to resolvin D1 suppressed self-limited murine peritonitis through reducing polymorphonuclear neutrophil recruitment and macrophage inflammatory cytokines $\mathrm{IL}-1$ and TNF-a production. ${ }^{23,24}$ Additionally, miR-219a-5p has been demonstrated to inhibit cancer cells proliferation, metastasis and epithelial-mesenchymal transition by targeting different oncogene. ${ }^{25-27}$ These findings support antiinflammatory and tumor suppressive roles of miR-219a-5p in the pathogenesis of diseases. However, whether and how miR-219a-5p regulates intestinal inflammatory response and pathogenesis of IBD remains elusive.

In the present study, we found that miR-219a-5p was significantly decreased in the inflamed intestinal mucosa and peripheral blood (PB)-CD4 ${ }^{+} \mathrm{T}$ cells of patients with IBD compared with healthy controls. Proinflammatory cytokines (e.g., IL-6, IL-12, IL-23 and TNF-a) stimulation significantly inhibited miR-219a-5p expression in PB$\mathrm{CD}^{+} \mathrm{T}$ cells in vitro. Lentivirus-mediated miR-219a-5p downregulation promoted $\mathrm{CD}_{4}{ }^{+} \mathrm{T}$ cells differentiate into Th1 and Th17 cells in IBD patients, whereas overexpression of miR-219a-5p had an opposite effect. Mechanistically, ETV5, a transcriptional factor that plays critical roles in regulating immune responses, was confirmed to be a functional target of miR-219a-5p in the pathogenesis of IBD. Overexpression of ETV5 facilitated Th1/Th17 immune response via upregulating the phosphorylation of STAT3 and STAT4. Furthermore, upregulation of miR-219a-5p ameliorated TNBS-induced colitis by suppressing Th1/Th17-mediated immune response. These data indicate that miR-219a-5p inhibits Th1/Th17-mediated immune responses in IBD. Supplementation of exogenous miR-219a-5p might provide a therapeutic approach for the treatment of IBD.

\section{RESULTS}

miR-219a-5p is decreased in the inflamed mucosa of IBD patients and TNBS-induced colitis mice

To investigate the expression and potential function of miR-219a$5 p$ in IBD, intestinal mucosa was obtained from healthy donors and inflamed site of IBD patients, and the pathological features of mucosa were shown in Supplemental Fig. 1a. Total RNA were isolated and the level of miR-219a-5p was detected by qRT-PCR. As shown in Fig. 1a, expression of miR-219a-5p was markedly decreased in the inflamed mucosa from CD and UC patients compared with that from healthy controls. We next compared the expression of miR-219a-5p in the inflamed mucosa and unaffected mucosa adjacent to inflamed site of the same patients with $C D$ or UC, and found that miR-219a-5p expression in the inflamed mucosa was lower than that of normal mucosa from the same IBD patients (Fig. 1b, c).

Since TNBS-induced intestinal mucosal inflammation is a wellestablished colitis model, we next compared miR-219a-5p expression in colon tissues from TNBS-induced colitis and control mice. Colorectal tissues were obtained from TNBS-induced colitis mice and control mice, and the histological features of colitis (colon transmural inflammation and hyperemia) were consistent with previous studies (Supplemental Fig. 1b). qRT-PCR analysis confirmed that colonic miR-219a-5p expression in TNBS-induced colitis mice was significantly downregulated compared with that in control mice (Fig. 1d). Taken together, these results suggest that miR-219a-5p is decreased in inflamed intestinal mucosa from both IBD patients and TNBS-induced colitis mice.

Proinflammatory cytokines IL-6, IL-12, IL-23 and TNF-a reduce miR219a-5p expression in CD4 ${ }^{+} \mathrm{T}$ cells

Although the exact pathogenesis of IBD remains elusive, $\mathrm{CD}^{+}{ }^{+} \mathrm{T}-$ cell-mediated inflammatory responses have been demonstrated to play crucial roles in the development of IBD. To further assess
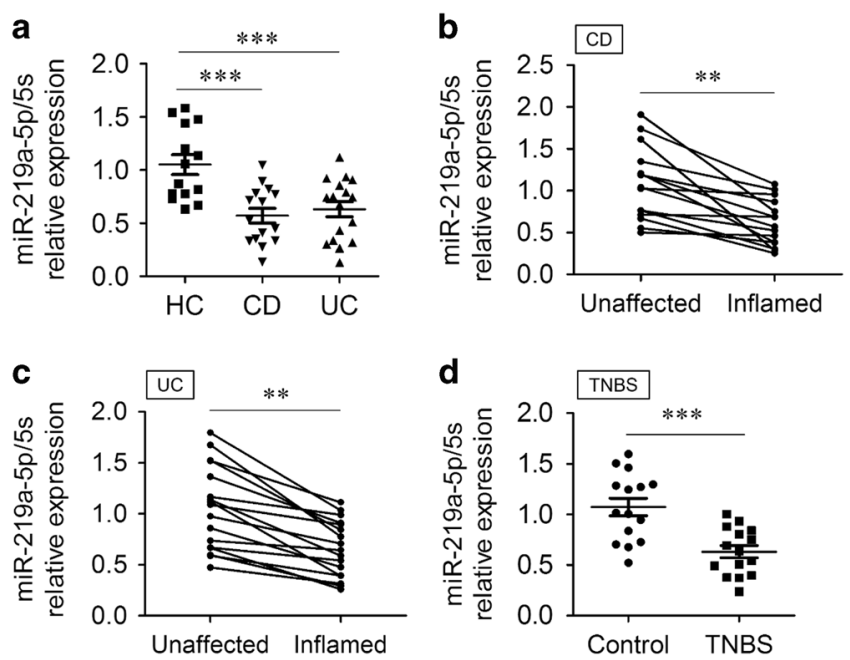

Fig. 1 miR-219a-5p is decreased in inflamed mucosa of IBD patients and TNBS-induced colitis mice. a Colon biopsies were obtained from colonic mucosa of healthy controls $(H C, n=14)$, inflamed intestinal mucosa of active CD (CD, $n=15)$ and UC (UC, $n=17)$. Expression of miR-219a-5p was detected by qRT-PCR using the snRNA $5 s$ as endogenous controls. ${ }^{* *} p<0.001$. b, $\mathbf{c}$ miR-219a- $p$ expression in unaffected and inflamed colonic mucosa from the same CD or UC patients was analyzed by qRT-PCR. ${ }^{* *} p<0.01$. d Colonic tissues were collected from TNBS-induced colitis mice $(n=15)$ and control mice $(n=15)$, and the expression of miR-219a-5p was measured by qRTPCR. ${ }^{* * *} p<0.001$. Data are representative of three independent experiments

the level of miR-219a-5p in IBD CD4 ${ }^{+} \mathrm{T}$ cells, CD4 ${ }^{+} \mathrm{T}$ cells were separated from the peripheral blood of patients with IBD and healthy controls for total RNA isolation. qRT-PCR analysis demonstrated that miR-219a-5p expression in peripheral blood (PB)-CD4 ${ }^{+} \mathrm{T}$ cells was significantly decreased in $\mathrm{CD}$ and UC patients compared with healthy controls (Fig. 2a). Accumulating evidences have demonstrated that the elevated proinflammatory cytokines (e.g., IL-1 $\beta$, IL-6, IL-23, and TNF- $\alpha$ ) contribute to the inflammatory immune response of IBD and certain miRNAs in $\mathrm{CD}^{+} \mathrm{T}$ cells have been confirmed to be regulated by these cytokines. ${ }^{28,29}$ We therefore investigated whether the increased proinflammatory mediators are responsible for the downregulation of miR-219a-5p in IBD. For this purpose, PB-CD4 ${ }^{+}$T cells were separated from healthy donors and activated with immobilized anti-CD3 and anti-CD28 mAs in vitro for $48 \mathrm{~h}$. These cells were then stimulated with IL-1 $\beta$, IL-6, IL-12, IL-23 and TNF- $a$ for a further $48 \mathrm{~h}$, respectively. qRT-PCR analysis revealed that IL-6, IL-12, IL-23 and TNF-a treatment markedly inhibited miR-219a-5p expression compared with that in cells cultured in medium alone (Fig. 2b). Consistently, similar results were found in $\mathrm{PB}-\mathrm{CD} 4^{+} \mathrm{T}$ cells isolated from CD and UC patients (Fig. 2c, d).

Previous studies demonstrated that signal transducers and activator of transcription 3 (STAT3) and nuclear factor kappa-B (NF-KB) are involved in IL-6-, IL-23- and TNF-a-induced miRNAs dysregulation. $^{30-32}$ To further investigate the mechanisms involved in IL-6-, IL-23- and TNF-a-induced miR-219a-5p downregulation, we selected specific STAT3 inhibitor (S3I-201) and NF$\mathrm{KB}$ inhibitor (BAY 11-7082) to treat PB-CD4 ${ }^{+} \mathrm{T}$ cells. As shown in Supplemental Fig. 2a and b, IL-6- or IL-23-mediated miR-219a-5p suppression was inverted by S3I-201. Moreover, treatment with BAY 11-7082 notably ameliorated the repressive effect on miR219a-5p expression induced by TNF-a (Supplemental Fig. 2c). These results indicate that STAT3 and NF-KB are involved in regulating miR-219a-5p expression under IL-6/IL-23 and TNF- $a$ exposure. 

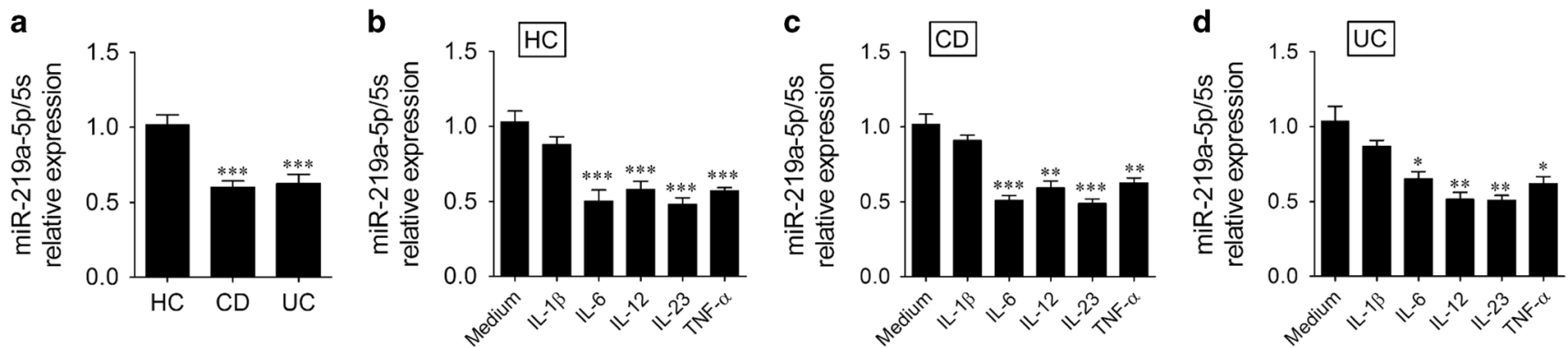

Fig. 2 Proinflammatory cytokines IL-6, IL-12, IL-23 and TNF- $\alpha$ reduce miR-219a-5p expression in CD4 ${ }^{+} \mathrm{T}_{\text {cells. a PB-CD4 }}{ }^{+} \mathrm{T}$ cells were obtained from healthy controls (HC, $n=12$ ), CD patients (CD, $n=8)$ and UC patients (UC, $n=10)$. The expression of miR-219a-5p was measured by qRTPCR. ${ }^{* * *} p<0.001$ versus HC. $\mathbf{b}-\mathbf{d}$ PB-CD4 ${ }^{+} \mathrm{T}$ cells isolated from $\mathrm{HC}(n=6), \mathrm{CD}(n=4)$ and UC $(n=4)$ were activated with plate-bound antihuman CD3 $(5 \mu \mathrm{g} / \mathrm{ml})$ and anti-human CD28 $(2 \mu \mathrm{g} / \mathrm{ml}) \mathrm{mAs}$ for $48 \mathrm{~h}$. These cells were then stimulated with IL-1 $\beta(20 \mathrm{ng} / \mathrm{ml})$, IL-6 $(40 \mathrm{ng} / \mathrm{ml})$, IL-12 $(20 \mathrm{ng} / \mathrm{ml}), \mathrm{IL}-23(40 \mathrm{ng} / \mathrm{ml})$ and TNF- $\alpha(20 \mathrm{ng} / \mathrm{ml})$ for $48 \mathrm{~h}$, respectively. miR-219a-5p expression was detected by qRT-PCR. ${ }^{*} p<0.05$, ${ }^{* *} p<0.01,{ }^{* * *} p<0.001$ versus cells cultured in medium alone. Data are representative of three independent experiments

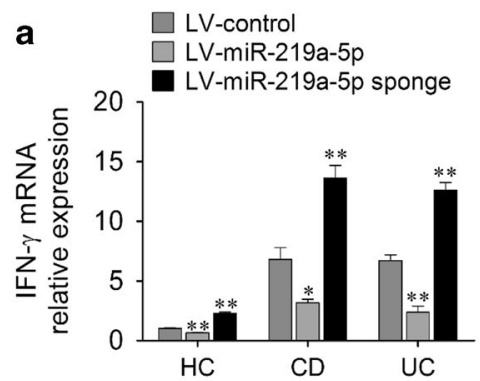

b

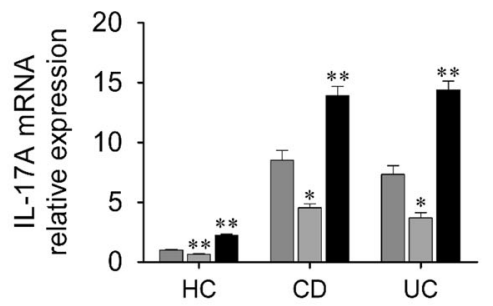

e

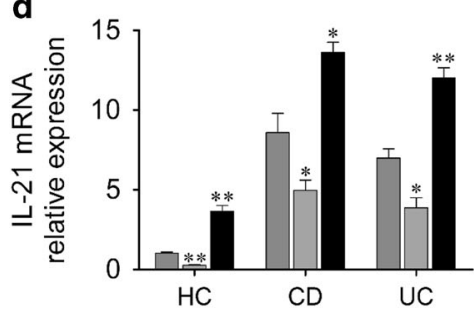

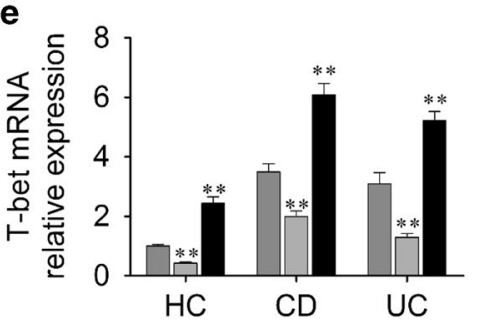

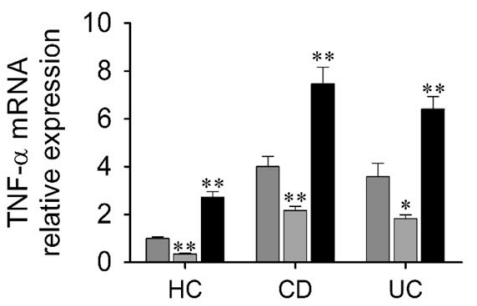

f

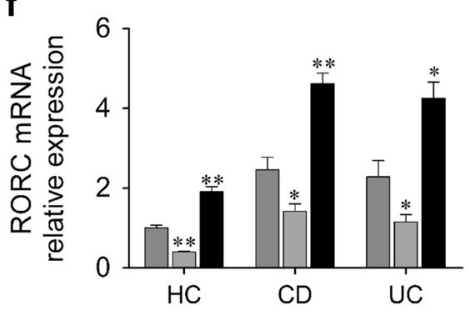

Fig. 3 Effect of miR-219a-5p on Th1/Th17 cell-related gene expression. PB-CD4 ${ }^{+}$T cells isolated from healthy controls $(\mathrm{HC}, n=6), \mathrm{CD}(\mathrm{CD}, n=$ 4) and UC (UC, $n=4)$ patients were activated with plate-bound anti-human CD3 $(5 \mu \mathrm{g} / \mathrm{ml})$ and anti-human CD28 $(2 \mu \mathrm{g} / \mathrm{ml}) \mathrm{mAs}$ for $48 \mathrm{~h}$, and then transduced with LV-miR-219a-5p, LV-miR-219a-5p sponge and LV-control for $6 \mathrm{~h}$, respectively. These cells were then cultured in medium for an additional 5 days. Total RNA was isolated from these cells and mRNA levels of IFN- $\gamma$ (a), IL-17A (b), TNF- $\alpha$ (c), IL-21 (d), T-bet (e) and RORC (f) were detected by qRT-PCR. ${ }^{*} p<0.05,{ }^{* *} p<0.01$ versus LV-control transduced CD4 ${ }^{+}$T cells. Data are representative of three independent experiments

miR-219a-5p inhibits Th1/Th17 cell differentiation in IBD It has been demonstrated that $\mathrm{CD}^{+}{ }^{+} \mathrm{T}$ cell-mediated immune response plays crucial roles in the pathogenesis of IBD. Considering that IL-6, IL-12 and IL-23 are crucial cytokines responsible for inducing Th1 and Th17 cell differentiation pathway, we then sought to investigate whether miR-219a-5p directly regulate the differentiation of $\mathrm{CD}^{+} \mathrm{T}$ cells. To this end, $\mathrm{PB}-\mathrm{CD} 4^{+} \mathrm{T}$ cells separated from patients with IBD and healthy donors were activated with platebound anti-human CD3 plus anti-human CD28 mAs for $48 \mathrm{~h}$. These cells were then transduced with Lentivirus-encoding miR-219a-5p (LV-miR-219a-5p) and miR-219a-5p-specific inhibitory oligonucleotide lentivirus (LV-miR-219a-5p sponge), respectively, which could increase or decrease endogenous miR-219a-5p expression. The validity of lentivirus transduction was confirmed by detecting miR219a-5p expression, which was significantly increased in LV-miR219a-5p-transduced cells but markedly decreased in LV-miR-219a-5p sponge transduced cells compared with cells transduced control lentivirus (Supplemental Fig. 3).

Total RNA was isolated for the analysis of mRNA levels of inflammatory cytokines (e.g., IFN- $\gamma$, IL-10, IL-17A, TNF- $\alpha$ and IL-21) and transcript factors (e.g., T-bet, RORC and Foxp3). As shown in
Fig. 3a-f, mRNA expression of IFN- $\gamma$, IL-17A, TNF- $a$, IL-21, T-bet and RORC were markedly decreased in LV-miR-219a-5p-transduced cells, whereas LV-miR-219a-5p sponge transduction exerted an opposite effect. However, no statistical difference was found in the expression of IL-10 and Foxp3 (Supplemental Fig. 4). The supernatants were also collected and protein levels of IFN- $\gamma$, IL$17 \mathrm{~A}$, TNF- $a$ and IL-21 were measured by ELISA. As shown in Fig. $4 a-d$, protein levels of IFN- $\gamma$, IL-17A, TNF- $a$ and IL-21 were markedly decreased in LV-miR-219a-5p transduced cells, but significantly increased in LV-miR-219a-5p sponge transduced cells compared with controls. Consistently, the percentage of IFN- $\gamma^{+}$ and IL-17A ${ }^{+} \mathrm{CD}^{+}{ }^{+}$cells was significantly decreased in LV-miR219a-5p transduced cells, but markedly increased in LV-miR-219a$5 p$ sponge transduced cells compared with controls (Fig. 4e, f). In addition, we detected whether miR-219a-5p could regulate $C D 4^{+}$ $\mathrm{T}$ cell apoptosis and proliferation in vitro. Flow cytometric analysis showed that miR-219a-5p overexpression and downregulation had no significant effect on $\mathrm{CD}^{+}{ }^{+} \mathrm{T}$ cell apoptosis (Supplemental Fig. 5) and proliferation (Supplemental Fig. 6). Collectively, these results suggest that miR-219a-5p suppresses Th1/Th17 cell differentiation. 

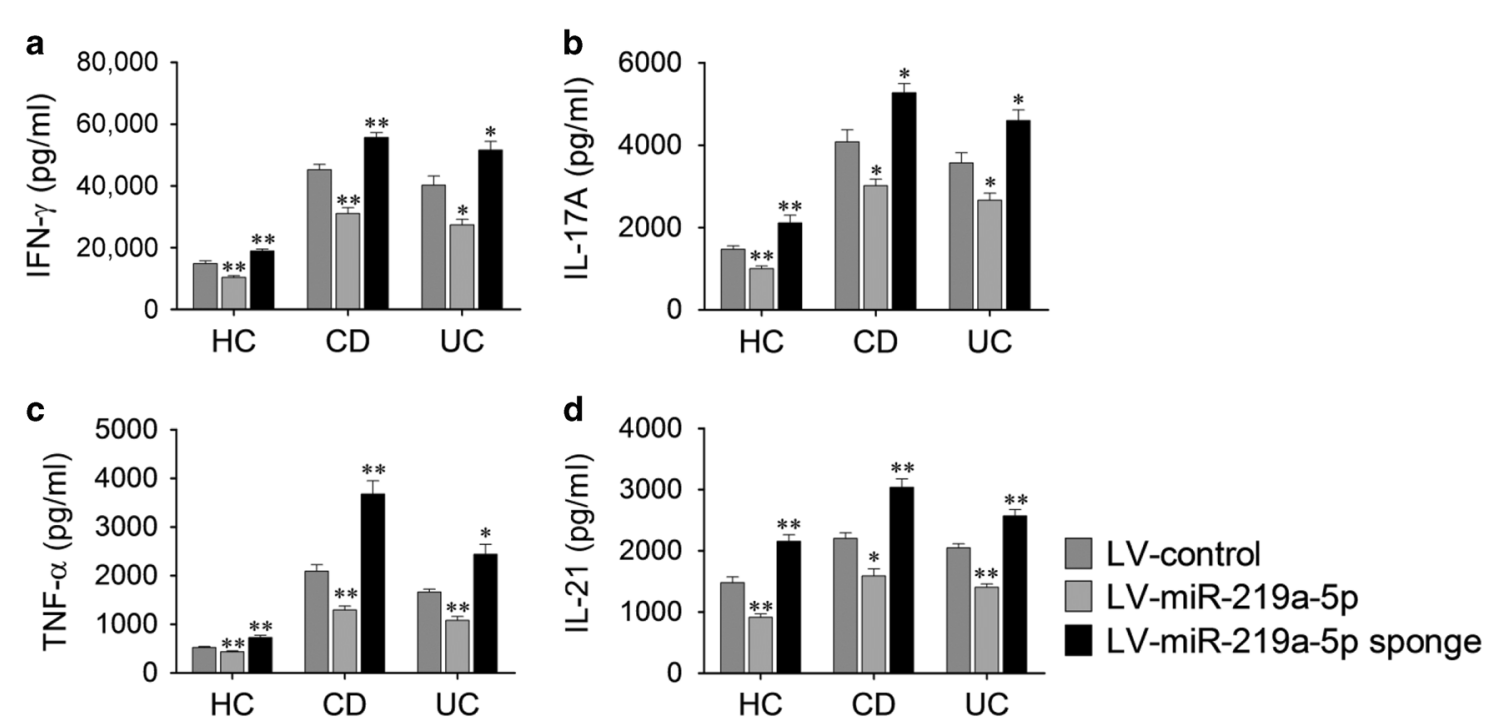

e

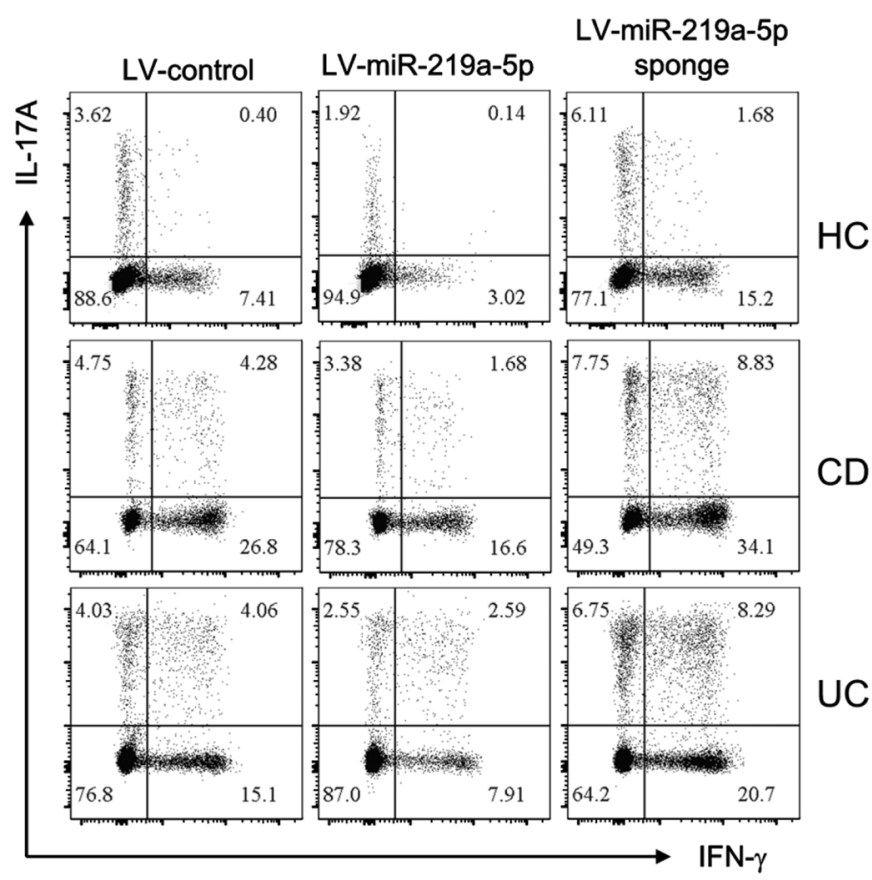

f

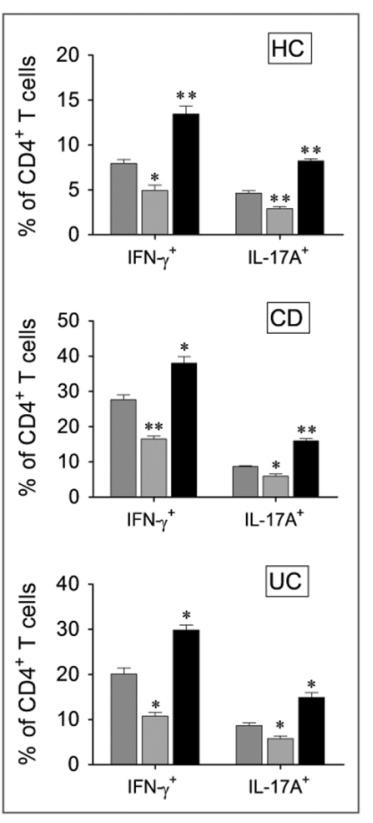

Fig. 4 miR-219a-5p suppresses Th1/Th17 cell immune response. PB-CD4 ${ }^{+}$T cells were transduced with LV-miR-219a-5p, LV-miR-219a-5p sponge or LV-control as described in Fig. 3. a-d Supernatants were harvested to detect protein levels of IFN- $\gamma$, IL-17A, IL-21 and TNF- $\alpha$ by

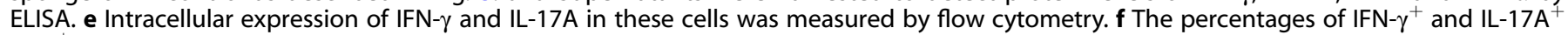
$\mathrm{CD}^{+} \mathrm{T}$ cells in HC (upper panel), patients with CD (middle panel) and UC (lower panel) are shown. ${ }^{*} p<0.05,{ }^{* *} p<0.01$ versus LVcontrol transduced $\mathrm{CD}^{+} \mathrm{T}$ cells. Data are representative of three independent experiments

ETV5 is a functional target of miR-219a-5p in CD4 ${ }^{+}$T cell of IBD miRNAs act as gene-silencers through suppressing mRNA transcription by binding to the $3^{\prime}$-untranslated (UTR) regions of the target gene. In an attempt to define how miR-219a-5p regulates Th1/Th17 response, we applied three bioinformatics prediction tools (TargetScan, microRNA.org and miRDB) to identify potential targets of miR-219a-5p that modulate intestinal inflammation. ETV5, a member of the ETS family of transcription factors, which has been identified to be associated with the pathogenesis of carcinoma and autoimmune diseases, ${ }^{33,34}$ exists potential binding sites of miR-219a-5p in the $3^{\prime}$-UTR (Fig. 5a). To confirm whether ETV5 mRNA is a direct target of miR-219a-5p in IBD, luciferase assays were performed by using the wild type (WT) and mutant ETV5 $3^{\prime}$-UTR. As illustrated in Fig. 5b, miR-219a-5p inhibited the activity of luciferase reporter with WT ETV5 $3^{\prime}$-UTR, but not that of the luciferase reporter containing a mutated $3^{\prime}$-UTR unable to bind to miR-219a-5p. To further confirm miR-219a-5p targeting ETV5 in IBD CD4 ${ }^{+}$T cells, ETV5 expression in LV-miR219a-5p and LV-miR-219a-5p sponge transduced cells were detected. We observed that LV-miR-219a-5p transduction markedly suppressed ETV5 expression compared with controls, whereas miR-219a-5p downregulation induced an opposite effect (Fig. 5c), indicating that miR-219a-5p directly downregulates ETV5 expression.

To investigate whether ETV5 is a functional target of miR-219a$5 p$ in the development of IBD, we next detected ETV5 expression in intestinal mucosa of IBD patients and healthy controls. Consistent with the decreased expression of miR-219a-5p, mRNA level of ETV5 was significantly increased in the inflamed mucosa of IBD patients compared with that in healthy controls (Fig. $5 \mathrm{~d}$ ). 


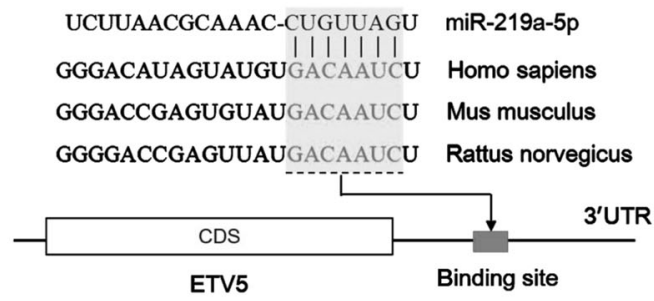

b

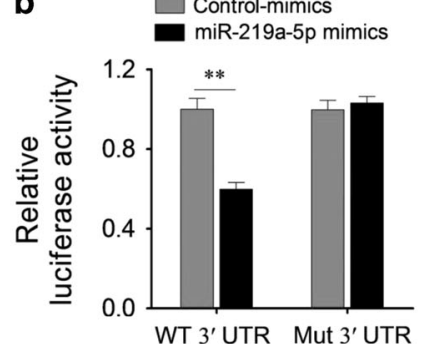

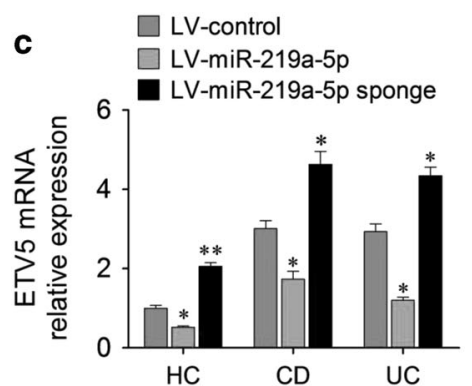

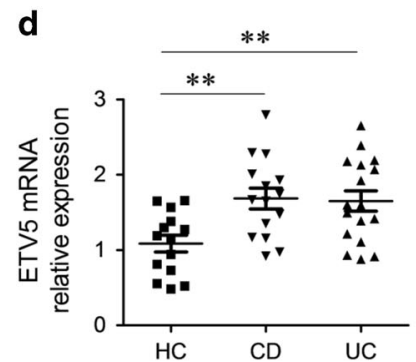

e.
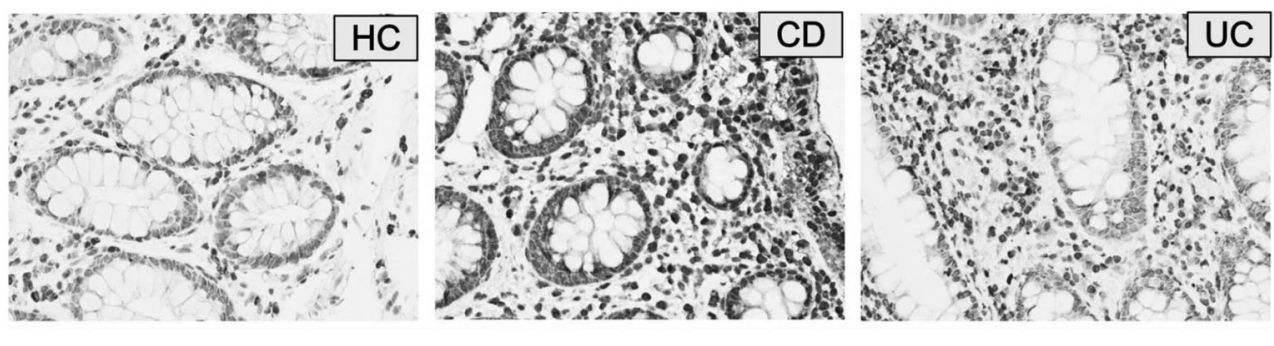

Fig. 5 miR-219a-5p targets ETV5 in IBD. a Schematic representation of conserved duplexes formed by the interaction of ETV5 $3^{\prime}$-UTR and miR219a-5p. The phylogenetically conserved miR-219a-5p binding site is marked in red. $\mathbf{b}$ The relative luciferase activity of a reporter containing a wild type (WT) or mutant (Mut) ETV5 3'-UTR in HEK-293 T cells was detected after cotransfected with miR-219a-5p mimics or control mimics for $24 \mathrm{~h}$. c Total RNA was extracted from CD4 ${ }^{+}$T cells as described in Fig. 3, and ETV5 expression was detected by qRT-PCR. ${ }^{*} p<0.05$, ${ }^{* *} p<0.01$ versus $\mathrm{LV}$-control transduced $\mathrm{CD} 4^{+} \mathrm{T}$ cells. d Colon biopsies were obtained from healthy controls (HC), CD and UC patients as mentioned in Fig. 1a, ETV5 expression was determined by qRT-PCR. ${ }^{* *} p<0.01$. e Representative colonic sections for ETV5 immunohistochemistry staining were obtained from healthy controls ( $\mathrm{HC}, n=10)$, patients with $\mathrm{CD}(\mathrm{CD}, n=8)$ and patients with $U C(\mathrm{UC}, n=10)$. Data are representative of three independent experiments

Similar results were observed in $\mathrm{PB}-\mathrm{CD}^{+}{ }^{+} \mathrm{T}$ cells isolated from patients with IBD and healthy controls (Supplemental Fig. 7). Furthermore, immunohistochemical staining revealed that ETV5positive cells in the lamina propria were markedly increased in the inflamed mucosa from patients with IBD (Fig. 5e), which is consistent with mRNA levels of ETV5 shown in Fig. $5 \mathrm{~d}$. Collectively, these results indicate that ETV5 is a functional target of miR-219a$5 p$ in $C D 4^{+} \mathrm{T}$ cell of IBD.

ETV5 promotes Th1/Th17 cell immune response through upregulating phosphorylation of STAT3 and STAT4

Considering that miR-219a-5p could inhibit Th1/Th17 cell differentiation and target ETV5, we further hypothesized that ETV5 might facilitate Th1/Th17 cell differentiation in IBD. To ascertain this, PB-CD4 ${ }^{+} \mathrm{T}$ cells isolated from IBD patients and healthy donors were transduced with lentivirus-expressing ETV5 (LV-ETV5), which could significantly increase the expression of ETV5 (Supplemental Fig. 8). As shown in Fig. 6a-d, enforced expression of ETV5 markedly upregulated the production of IFN- $\gamma$, IL-17A, TNF- $a$ and IL-21 in PB-CD4 ${ }^{+}$T cells. Consistently, the percentage of IFN $-\gamma^{+}$and IL-17A ${ }^{+}$CD4 ${ }^{+}$T cells was significantly increased in LVETV5-transduced cells compared with controls (Supplemental Fig. 9). In addition, qRT-PCR analysis demonstrated that ETV5 overexpression significantly increased mRNA levels of IFN- $\gamma$, IL17A, TNF-a and IL-21 (Fig. 6e-h), which is consistent with our findings in miR-219a-5p. However, mRNA expression of T-bet and RORC was not affected by ETV5 upregulation (Supplemental Fig. 10).

Consistent with the significant suppression of miR-219a-5p expression in cytokine-treated $\mathrm{CD}^{+}{ }^{+} \mathrm{T}$ cells (Fig. 2b), we also found simultaneous induction of ETV5 expression in IL-6-, IL-12-, IL-23- or TNF-a-stimulated cells (Supplemental Fig. 11). Previous studies reported that IL-12-induced phosphorylation of STAT4 ( $p$ STAT4) and IL-6/IL-23-activated p-STAT3 contribute to Th1 and Th17 cell differentiation, respectively. ${ }^{35-38}$ We then sought to investigate whether ETV5 regulates Th1 and Th17 cell differentiation through p-STAT4 and p-STAT3 signal pathway. To this end, protein levels of p-STAT3 and p-STAT4 in PB-CD4 ${ }^{+}$T cells transduced with LV-ETV5 or LV-control were detected by western blot. As shown in Supplemental Fig. 12a, ETV5 overexpression significantly increased the protein levels of p-STAT3 and p-STAT4. However, upregulation of ETV5 had no significant effect on mRNA levels of STAT3 and STAT4 (Supplemental Fig. 12b, c). In addition, we observed that LV-miR-219a-5p sponge transduction significantly increased the protein level of ETV5 coincided with upregulation of $\mathrm{p}$-STAT3 and p-STAT4 in PB-CD4 ${ }^{+} \mathrm{T}$ cells (Supplemental Fig. 13). These data imply that p-STAT3 and pSTAT4 are involved in the ETV5 and miR-219a-5p regulation of Th1/Th17 cell differentiation.

miR-219a-5p ameliorates intestinal inflammatory response in TNBS-induced colitis in mice

TNBS-induced colitis is a well-established experimental colitis model, which is associated with increased Th1/Th17-mediated intestinal inflammatory immune response in the colon. ${ }^{39-41}$ Recent studies demonstrated that exogenous supplementation of miRNA exerted therapeutic effect in the development of colitis in vivo. ${ }^{42-44}$ Since miR-219a-5p overexpression decreased Th1/ Th17 immune response in vitro, we then asked whether exogenous miR-219a-5p could reduce intestinal inflammation in vivo. To this end, the in vivo-jetPEl-mediated miR-219a-5p precursor was injected intraperitoneally into mice as described in the Methods section. As shown in Supplemental Fig. 14, administration of miR-219a-5p precursor markedly upregulated the colonic miR-219a-5p expression compared with control precursor treatment. We found that supplementation of miR-219a-5p markedly ameliorated TNBS-induced colitis, characterized by less body weight loss, moderate colon shortening, slight histological damage and low pathologic scores (Fig. 7a-e).

We next assessed whether miR-219a-5p overexpression could affect the Th1/Th17 cell-related inflammation response in vivo. For this, colonic lamina propria cells were isolated and the phenotype 

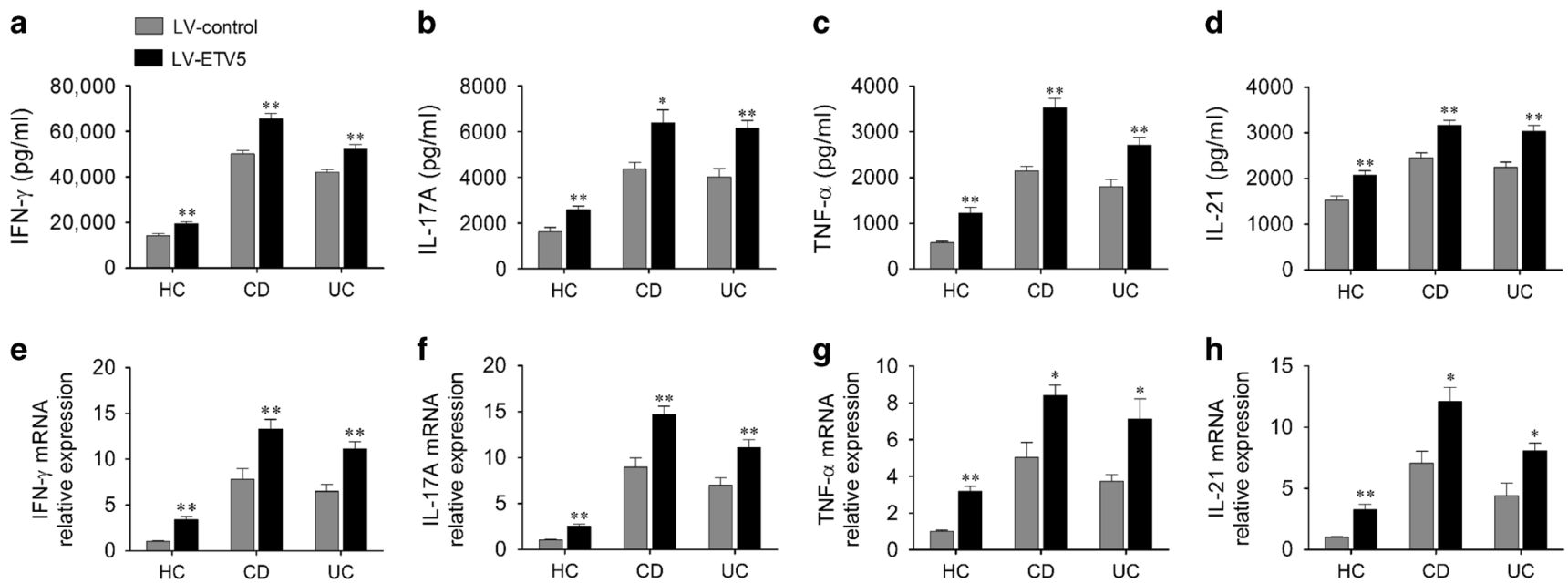

Fig. 6 ETV5 promotes Th1/Th17 cell immune response. PB-CD4 ${ }^{+}$T cells separated from healthy controls $(H C, n=5), C D$ patients $(C D, n=3)$, and UC patients (UC, $n=4)$ were activated with plate-bound anti-human CD3 $(5 \mu \mathrm{g} / \mathrm{ml})$ and anti-human CD28 $(2 \mu \mathrm{g} / \mathrm{ml}) \mathrm{mAbs}$ for $48 \mathrm{~h}$, and then transduced with lentivirus-expressing ETV5 (LV-ETV5) or scrambled control lentivirus (LV-control). a-d Supernatants were collected and the protein levels of IFN- $\gamma(\mathbf{a})$, IL-17A (b), TNF- $\alpha$ (c) and IL-21 (d) were detected by ELISA. $\mathbf{e}-\mathbf{h}$ CD4 ${ }^{+}$T cells were harvest for total RNA isolation and the mRNA levels of IFN- $\gamma(\mathbf{e})$, IL-17A (f), TNF- $\alpha$ (g) and IL-21 (h) were measured by qRT-PCR. ${ }^{*} p<0.05,{ }^{* *} p<0.01$ versus LVcontrol transduced cells. Data are representative of three independent experiments

of $\mathrm{CD}^{+} \mathrm{T}$ cells were detected by flow cytometry. We observed that miR-219a-5 precursor treatment decreased the percentage of IFN $-\gamma^{+} \mathrm{CD}^{+} \mathrm{T}$ cells (Fig. 8a) and IL-17A ${ }^{+} \mathrm{CD}^{+} \mathrm{T}$ cells (Fig. 8b) in the TNBS-exposed mice. Furthermore, we also found that colonic IFN- $\gamma$, IL-17A, TNF- $a$, IL-21, T-bet and RORyt expression was significantly decreased in miR-219a-5p precursor administrated mice compared to that from control precursor treated counterparts under exposure to TNBS (Fig. 8c-h), consistent with our findings in Figs. 3 and 4 . These findings thus demonstrate that miR-219a-5p suppresses intestinal inflammation through inhibiting Th1/Th17 cell immune responses in colitis.

\section{DISCUSSION}

Th1/Th17-mediated inflammatory responses play critically roles in the pathogenesis of IBD, and functional regulators of Th1/Th17 cell differentiation are regarded to possess potential values in the clinical diagnosis and treatment of this complex immune disease. ${ }^{45,46}$ IL-12 contributes to Th1 cells differentiation though activating STAT4 pathway. ${ }^{35,47}$ IL-6- and IL-23-activated phosphorylation of STAT3 is a critical and specific factor required for the generation and stabilization of Th17 cells. ${ }^{36-38}$ Although Th1/Th17 cell differentiation is considered to be regulated by special cytokines and transcription factors, the function of miRNA pathways that essentially control the differentiation of Th1/Th17 cells remains unclear. To date, a number of miRNAs have been identified to be involved in the development and pathogenesis of $I_{B D}{ }^{48}$ whereas roles of miR-219a-5p in the pathogenesis of IBD have not been reported. In the present study, we demonstrated that miR-219a-5p expression is significantly decreased in the inflamed mucosa from patients with IBD and TNBS-induced colitis mice. miR-219a-5p inhibits Th1/Th17-cell-mediated immune responses and negatively regulates the pathogenesis of IBD.

Accumulating evidence has demonstrated that proinflammatory cytokine (e.g., IL-1 $\beta, \mathrm{IL}-6, \mathrm{IL}-12, \mathrm{IL}-23$ and TNF- $\alpha$ )-induced aberrant miRNAs expression contributes to Th1/Th17-related mucosal inflammation in IBD. ${ }^{28,29}$ A recent study indicated that TNF-a promotes miR-301a expression in patients with IBD, and this effect is successfully reversed by anti-TNF-a therapy. ${ }^{28}$ miR-301a promotes Th17 cell differentiation and TNF-a production both in vivo and in vitro. miR-10a was found to be decreased in the inflamed mucosa of IBD and suppresses Th1/Th17 cell immune responses. ${ }^{14}$ Here, we identified that miR-219a-5p expression is significantly decreased in the inflamed intestinal mucosa and PB$\mathrm{CD}^{+} \mathrm{T}$ cells from patients with IBD. Further investigation demonstrated that recombinant IL-12, IL-6, IL-23 and TNF-a could decrease miR-219a-5p expression in PB-CD4 ${ }^{+}$T cells, implying that miR-219a-5p downregulation might be the consequence of intestinal inflammation. Importantly, silencing miR-219a-5p significantly increased the expression of INF- $\gamma$, IL-17A, T-bet and RORC in $\mathrm{CD}^{+} \mathrm{T}$ cells from IBD patients, whereas miR-219a-5p overexpression exerted opposite effects. These findings indicate that inflammation-induced miR-219a-5p downregulation contributes to Th1/Th17 differentiation in IBD.

Multiple miRNAs have been demonstrated to play critical roles in the pathogenesis of TNBS-induced colitis through affecting Th1/ Th17-mediated colonic inflammation response, and possess valuable potential in the clinical applications of colitis. ${ }^{9,28,49} \mathrm{~A}$ previous report indicated that miR-141 expression is decreased in the inflamed mucosa of CD patients and experimental colitis mice. Supplementation of miR-141 ameliorated the severity of TNBSinduced colitis through targeting CXCL12 $\beta$ in vivo. ${ }^{42}$ miR-31 expression was increased in the inflamed intestinal mucosa of patients with $C D$ and colitic mice. Overexpression of miR-31 aggravated TNBS-induced colitis and IL-10 knockout spontaneous colitis through promoting Th1/Th17-mediated intestinal inflammation response, whereas administration of miR-31 inhibitor exerted a therapeutic effect in the development of colitis. ${ }^{9} \mathrm{~A}$ recent study confirmed that in vivo delivery of miR-219a-5p promotes myelin repair and improves the clinical symptoms in animal models of multiple sclerosis $(\mathrm{MS}){ }^{50}$ suggesting the important clinical relevance of miR-219a-5p in the treatment of MS. In this study, we demonstrated that exogenous supplementation of miR-219a-5p could ameliorate TNBS-induced mucosal inflammation and inhibit IFN- $\gamma^{+} / \mathrm{IL}^{-17 \mathrm{~A}^{+}} \mathrm{CD} 4^{+} \mathrm{T}$ cell infiltration. These findings indicate that miR-219a-5p plays a protective role in the development of colitis through suppressing Th1/Th17mediated inflammation response.

ETV5, a member of the ETS family, has been reported to be significantly associated with numerous progression of carcinoma and autoimmune diseases. ${ }^{33,34}$ In the present study, our findings confirmed that ETV5 is a functional target of miR-219a-5p and is significantly increased in the inflamed mucosa and PB-CD4 ${ }^{+}$ $T$ cells of IBD patients. Enforced expression of ETV5 significantly 

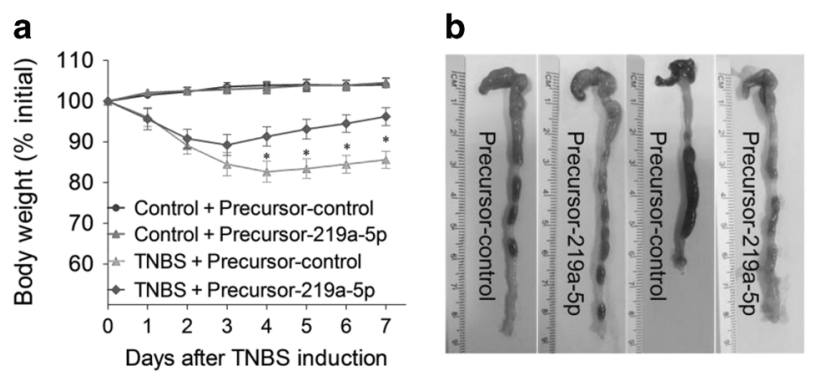

d

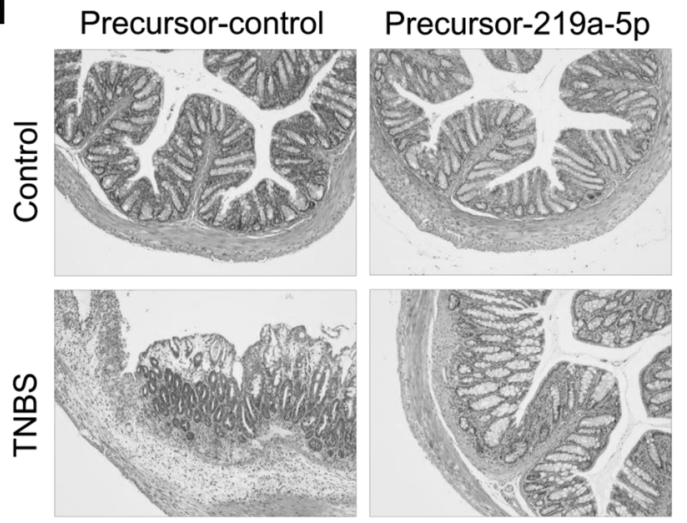

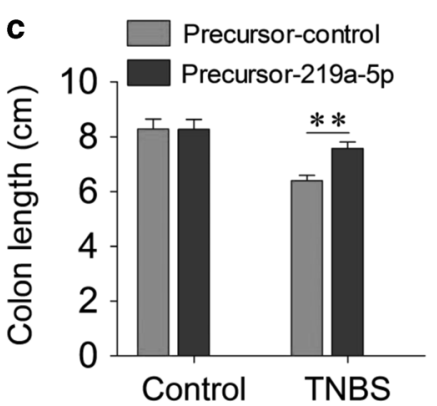

e

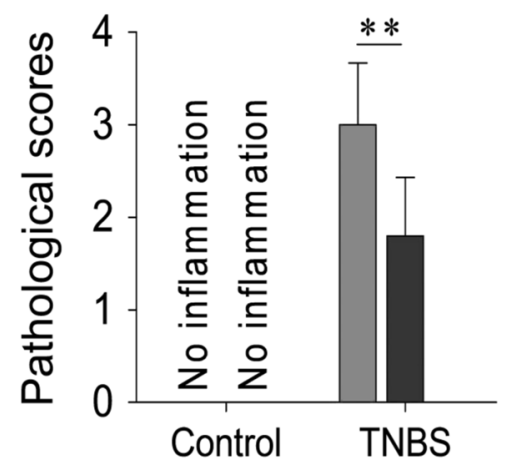

Fig. 7 Administration of miR-219a-5p precursor ameliorates TNBS-induced colitis. Mice treated with TNBS or alcohol (Control) were administrated with miR-219a-5p precursor (Precursor-219a-5p) or control precursor (Precursor-control) as described in the Methods section. a The changes of body weight were measured daily. Gross morphology (b) and length (c) of the colons on day 7 after TNBS induction are shown. d Representative H\&E staining of colonic sections from mice in each group. e The changes in histological scores from colorectal sections were calculated as indicated. Data represent means \pm SEM from three independent experiments. ${ }^{* *} p<0.01$

increased the expression of IL-17A, IFN- $\gamma$, TNF- $a$ and IL-21 in PB$\mathrm{CD}^{+} \mathrm{T}$ cells, which is consistent with our observation in miR219a-5p. These findings reveal that downregulated miR-219a-5p and increased ETV5 promote inflammation response through regulating Th1/Th17-mediated inflammatory responses (Supplemental Fig. 15). Interestingly, our findings demonstrated that miR219a-5p inhibits T-bet and RORC expression in human CD4 T cells, whereas ETV5 overexpression had no effect on T-bet and RORC expression, suggesting that miR-219a-5p probably targets other genes in addition to ETV5 in regulating $\mathrm{CD}^{+}{ }^{+} \mathrm{T}$ cell differentiation.

Previous studies indicate that ETV 5 augments IFN- $\gamma$ production in mice Th1 cells through influencing the promoter of IFN- ${ }^{51,52}$ Duy Pham et al. ${ }^{34}$ have demonstrated that EVT5 is a positive regulator of Th17 cell differentiation and is responsible for the pathogenesis of allergen-induced airway inflammation in mice. As a transcription factor, mice ETV5 gene promotes Th17 cell differentiation through directly binding to the promoter of IL17A. However, bioinformatics analysis showed that human ETV5 gene has no binding site in the promoter region of IFN- $\gamma$ and IL17A gene, implying the existence of other signaling pathways for ETV5 regulated Th1/Th17 differentiation. It has been demonstrated that IL-12-induced p-STAT4 activation and IL-6/IL-23activated $\mathrm{p}$-STAT3 contribute to Th1 and Th17 cell differentiation, respectively. ${ }^{35-38}$ In this study, we found that protein levels of $p$ STAT3 and p-STAT4 were significantly increased in ETV5 overexpressed PB-CD4 ${ }^{+}$T cells, as well as in LV-miR-219a-5p sponge transduced cells. These data imply that p-STAT3 and p-STAT4 are involved in the ETV5 and miR-219a-5p regulation of Th1/Th17 cell differentiation.

To summarize, our data demonstrate that miR-219a-5p is of great importance in protecting against intestinal mucosal inflammation. miR-219a-5p inhibits Th1/Th17-mediated intestinal inflammatory response both in vitro and in vivo. Thus, our study provides a new light on the function of miR-219a-5p in the regulation of IBD, and supplementation of exogenous miR-219a$5 \mathrm{p}$ might be a promising approach for the treatment of IBD.

\section{METHODS}

Patients and samples

Colonoscopic biopsies were obtained from inflamed and unaffected areas of the colons of 15 active CD patients, 17 active UC patients and 14 healthy controls. EDTA anticoagulated peripheral blood samples (approximately $25 \mathrm{ml}$ ) were obtained from 15 active CD patients, 18 active UC patients and 23 healthy volunteers. The clinical characteristics of IBD patients are shown in Supplementary Table 1. All patients and healthy controls enrolled in this study were recruited from the Department of Gastroenterology, Affiliated People's Hospital of Jiangsu University (Zhenjiang, China), from November 2016 to June 2019. The diagnosis for CD and UC patients was confirmed by clinical features, endoscopic examination and histological findings after exclusion of other autoimmune diseases, infectious diseases and tumors. Diseases severity was assessed according to Crohn's disease activity index (CDAl) for CD patients and Mayo index for UC patients. This study was approved by the Institutional Review Board for Clinical Research of the Affiliated People's Hospital of Jiangsu University. Written informed consent was obtained from each participant before the study.

Establishment of TNBS-induced colitis

Six to eight weeks female BALB/c mice were purchased from the Laboratory Animal Center of Jiangsu University (Jiangsu, China). All animal experiments in this research were approved by the Animal Care and Use Committee of Jiangsu University. All animals received care according to Chinese legal requirements. TNBS (Sigma-Aldrich, St. Louis, MO, USA)-induced acute colitis in mice was performed as 
a Precursor-control Precursor-219a-5p

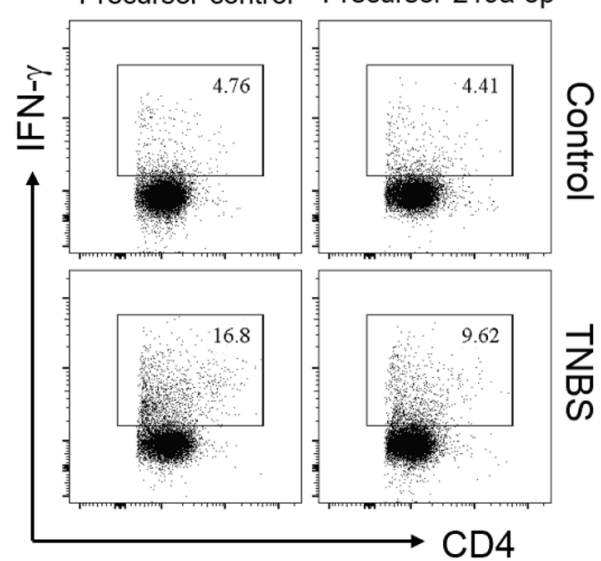

b

Precursor-control Precursor-219a-5p

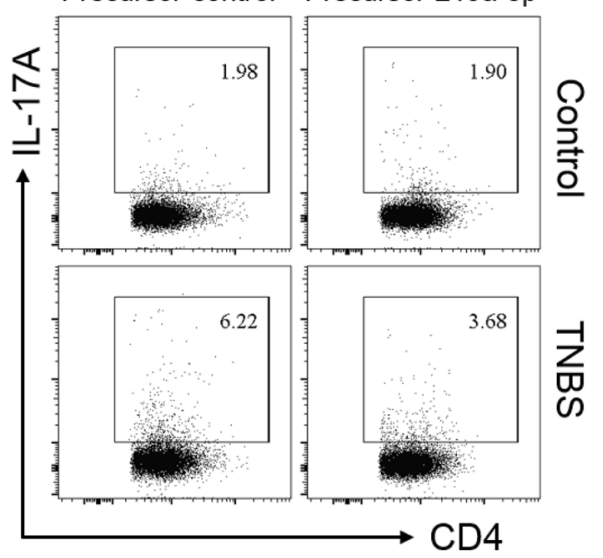

\section{Precursor-control Precursor-219a-5p}

C

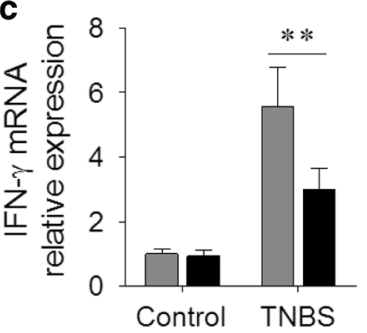

f

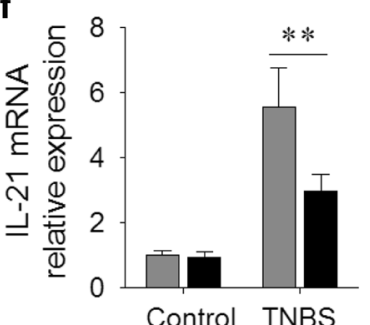

d

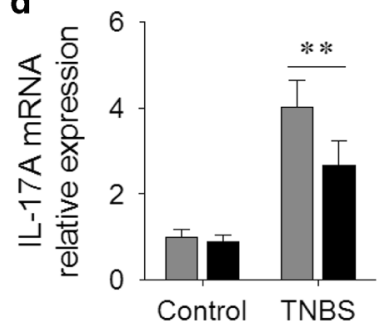

g

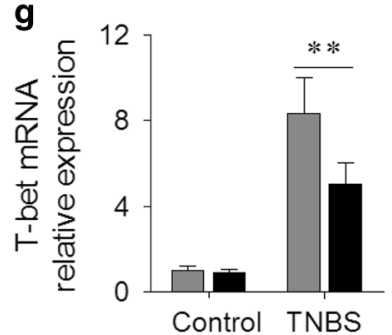

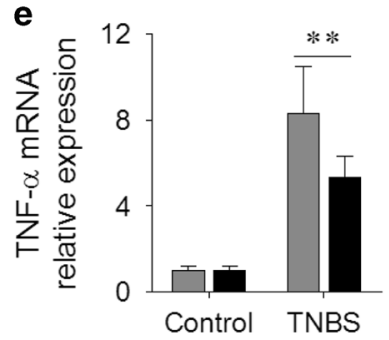

h

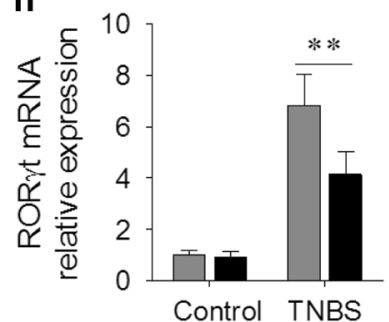

Fig. 8 miR-219a-5p suppresses Th1/Th17 cell immune response in TNBS-induced colitis in mice. Colonic tissues were collected from mice as described in Fig. 7. Lamina propria cells were isolated and the percentage of IFN- $\gamma^{+}$CD4 ${ }^{+} \mathrm{T}$ cells (a) and IL-17A ${ }^{+} \mathrm{CD} 4^{+} \mathrm{T}$ cells (b) was detected by flow cytometry. c-h Colonic IFN- $\gamma$ (c), IL-17A (d), TNF- $\alpha$ (e), IL-21 (f), T-bet (g) and ROR $\gamma \mathrm{t}$ (h) mRNA expression was detected by qRT-PCR. Data represent means \pm SEM from three independent experiments. ${ }^{* *} p<0.01$

reported previously. ${ }^{39,41}$ Briefly, after anesthetization, $150 \mu \mathrm{l}$ of $2.5 \%$ TNBS (mixing $5 \%(\mathrm{w} / \mathrm{v})$ TNBS with equal volume of absolute ethanol) was injected into the colon lumen $4 \mathrm{~cm}$ from the anus via a polyethylene catheter. Mice were then held in a vertical position for at least $30 \mathrm{~s}$ to avoid TNBS solution excretion. Control mice were administered intracolonically with equal volume of $50 \%$ ethanol alone. After 7 days, colorectal tissues were collected and the pathological features of colitis were analyzed by colon $\mathrm{H} \& \mathrm{E}$ staining. Total RNA was isolated from colonic tissues of TNBSinduced colitis mice and control mice, and the expression of miR-219a-5p was detected by qRT-PCR.

Peripheral blood $\mathrm{CD}^{+}{ }^{+}$T-cell purification and activation PBMCs were separated from peripheral blood samples of IBD patients and healthy controls using Ficoll (GE Healthcare; Uppsala, Sweden) gradient centrifugation. $\mathrm{CD}^{+} \mathrm{T}$ cells were then purified by anti-human CD4 Particles (BD Biosciences, San Diego, CA, USA) from PBMCs according to the manufacturer's protocol. These cells $\left(5 \times 10^{5} /\right.$ well) were cultured in complete Roswell Park Memorial Institute (RPMI)-1640 medium (WISENT, Canada) supplemented with sodium pyruvate ( $1 \mathrm{mM}$; Gibco, Life Technologies, Carlsbad, California, USA), 10\% fetal bovine serum (WISENT), streptomycin $(100 \mathrm{mg} / \mathrm{ml})$ and penicillin $(100 \mathrm{U} / \mathrm{ml}), 2$-mercaptoethanol $(50 \mu \mathrm{M}$; MP Biomedicals, Santa Ana, California, USA) and 4-(2-hydroxyethyl) piperazine-1-ethanesulfonic acid (HEPES), and preactivated with plate-bound anti-human CD3 $(5 \mu \mathrm{g} / \mathrm{ml})$ and antihuman CD28 $(2 \mu \mathrm{g} / \mathrm{ml})$ mAbs. After $48 \mathrm{~h}$, these cells were harvested for lentivirus-mediated $\mathrm{CD}^{+}{ }^{+} \mathrm{T}$ cell transduction and proinflammatory cytokines (e.g., IL-1 $\beta$, IL-6, IL-12, IL-23, and TNF-a) stimulation assays.

Lentivirus-mediated $\mathrm{CD}^{+} \mathrm{T}$ cell transduction To investigate the roles of miR-219a-5p, CD4 ${ }^{+} \mathrm{T}$ cells isolated from IBD patients and healthy controls were cultured in complete RPMI1640 medium and stimulated with plate-bound anti-human CD3 and anti-human CD28 mAbs for $48 \mathrm{~h}$. These cells were then transduced with Lentivirus-encoding miR-219a-5p (LV-miR-219a$5 p)$, miR-219a-5p-specific inhibitory oligonucleotide lentivirus (LVmiR-219a-5p sponge), and scrambled control lentivirus (LVcontrol), respectively. Briefly, preactivated $\mathrm{CD}^{+} \mathrm{T}$ cells $\left(1 \times 10^{5}\right.$ / 
well) were seeded into a new 24-well plate and co-cultured with LV-miR-219a-5p, LV-miR-219a-5p sponge or LV-control $(\mathrm{MOI}=$ 100). The 24-well plate was placed in a flat angle centrifuge and centrifuged at $1000 \times g$ for $90 \mathrm{~min}$ at room temperature, and then cultured in a $5 \% \mathrm{CO}_{2}$ incubator at $37^{\circ} \mathrm{C}$ for $6 \mathrm{~h}$. After transduction, these cells continued to be cultured in fresh medium for 5 days. $\mathrm{CD}^{+}{ }^{\mathrm{T}}$ cells were then collected for total RNA extraction, and the mRNA levels of IFN- $\gamma$, IL-17A, IL-21, TNF- $a$, T-bet, and RORC were detected by qRT-PCR. Moreover, the supernatants were also harvested for the protein level of IFN- $\gamma$, IL-17A, IL-21 and TNF- $a$ measurement. Lentivirus-encoding ETV5 (LV-ETV5) transfection has a similar process to miR-219a-5p.

Total RNA isolation and qRT-PCR

Total RNA was extracted from colonic tissues and CD4 ${ }^{+} \mathrm{T}$ cells with TRIzol reagent (Ambion, Life Technologies; Carlsbad, CA, USA). For miR-219a-5p expression analysis, reverse transcription primer and a pair of qPCR primers were designed by RiboBio (Guangzhou, China). Complementary DNA (CDNA) was generated by riboSCRIPT ${ }^{\mathrm{TM}}$ Reverse Transcription Kit (RiboBio) and qRT-PCR was performed in the 7500HT Fast Real-Time PCR System (Applied Biosystems; Carlsbad, CA, USA) using Bulge-Loop ${ }^{T M}$ miRNA qRT-PCR Starter Kit (RiboBio) according to the manufacturer's protocol. ROX dye (Thermo Fisher) was used to correct fluorescence and the expression levels of miR-219a-5p were normalized to snRNA $5 \mathrm{~s}$. To analyze the mRNA levels of IFN- $\gamma$, IL-17A, IL-21, TNF- $a$, T-bet, and RORC, reverse transcription was performed using $5 \times$ PrimeScript RT Master Mix Kit (TaKaRa, Dalian, Chnia). qRT-PCR was then carried out with TB Green $^{\text {TM }}$ Premix Ex Taq ${ }^{\text {TM }}$ Kit in the $7500 \mathrm{HT}$ Fast Real-Time PCR System (Applied Biosystems) and GAPDH was used as a housekeeping gene. Each example was performed in triplicate wells and the expression levels of genes were analyzed by the $2^{-\Delta \Delta C T}$ method. The primers used are listed in Supplementary Table 2.

\section{ELISA}

The protein levels of IFN- $\gamma$, IL-10, IL-17A, IL-21 and TNF- $a$ in the culture supernatants of lentivirus-transduced $\mathrm{CD}^{+}{ }^{+} \mathrm{T}$ cells were detected by ELISA kits (BioLegend; San Diego, CA, USA) according to the manufacturer's protocol.

\section{Flow cytometry}

$\mathrm{CD}^{+}{ }^{+}$T cells were stimulated with ionomycin $(750 \mathrm{ng} / \mathrm{ml}$, SigmaAldrich), PMA ( $50 \mathrm{ng} / \mathrm{ml}$, Sigma-Aldrich) and brefeldin A solution ( $1 \mathrm{mg} / \mathrm{ml}$, eBioscience) in complete RPMl-1640 medium for $5 \mathrm{~h}$. Cells were collected and stained with cell-surface antibodies to human CD4 for $30 \mathrm{~min}$ at $4{ }^{\circ} \mathrm{C}$. Meanwhile, Live/Dead Fixable Dead Cell Stain Kit (Invitrogen, Life Technologies; Eugene, Oregon, USA) was used to exclude dead cells. After surface staining, these cells were fixed and permeabilized in Fixation/Permeabilization solution (eBioscience) for $30 \mathrm{~min}$ at $4{ }^{\circ} \mathrm{C}$. Subsequently, IFN- $\gamma$ (BioLegend; San Diego, CA, USA) and IL-17A (BioLegend) intracellular staining was carried out according to the manufacturer's instructions. Finally, all samples were processed on a flow cytometer (BD FACS Canto II), and data were analyzed using the FlowJo software.

Treatment and assessment of TNBS-induced colitis To investigate the potential role of miR-219a-5p in TNBS-induced colitis in vivo, the in vivo-jetPEl-mediated miR-219a-5p precursor was injected intraperitoneally into mice, as described previously. ${ }^{9,42,53}$ Briefly, miR-219a-5p precursor $(5 \mathrm{mg} / \mathrm{kg})$ or control precursor were administrated to mice for 4 consecutive day $12 \mathrm{~h}$ after $150 \mu \mathrm{l}$ 2.5\% TNBS injection. Non-TNBS-treated mice were injected with miR-219a-5p precursor or control precursor as negative controls. The body weight was evaluated daily. Mice were sacrificed on day 7 after TNBS induction and the colons were collected for macroscopic observation, histopathological detection, the phenotype of $\mathrm{CD}^{+} \mathrm{T}$ cell analysis and proinflammatory cytokine expression measurement. For histopathological examination, paraffin-embedded colon tissues were stained with $\mathrm{H} \& \mathrm{E}$, and pathological score was performed according to a scoring system described previously. ${ }^{39}$

\section{Statistical analysis}

Data were expressed as mean \pm SEM from three independent experiments. Statistical analysis was carried out using Prism 5.0 (GraphPad Software; San Diego, CA, USA). Statistical comparisons were performed using a paired Student's $t$ test, unpaired twotailed Student's $t$ test, and one-way analysis of variance (ANOVA). $p<0.05$ represented statistical significance.

\section{ACKNOWLEDGEMENTS}

This work was supported by grants from the National Natural Science Foundation of China (81700494), Jiangsu Provincial Key Research and Development Program (BE2017692) and supporting funds from Zhenjiang Municipal Health Commission and Affiliated People's Hospital of Jiangsu University (K201733, K201741).

\section{AUTHOR CONTRIBUTIONS}

Y.S. conceived the project and wrote the manuscript. Y.X. and J.Y. supervised the experimental work and revised the manuscript; Y.S., C.Q., Y.Z. and T.W. performed the experiments and data analysis. Y.X., C.X., and S.D. diagnosed the patients, recorded the consent and provided clinical samples and information.

\section{ADDITIONAL INFORMATION}

The online version of this article (https://doi.org/10.1038/s41385-019-0216-7) contains supplementary material, which is available to authorized users.

Competing interests: The authors declare no competing interests.

Publisher's note Springer Nature remains neutral with regard to jurisdictional claims in published maps and institutional affiliations.

\section{REFERENCES}

1. Shi, Y. et al. Smad nuclear interacting protein 1 (SNIP1) inhibits intestinal inflammation through regulation of epithelial barrier function. Mucosal Immunol. 11, 835-845 (2018).

2. Ma, C. et al. Critical role of CD6highCD4+ T cells in driving Th1/Th17 cell immune responses and mucosal inflammation in IBD. J. Crohn's Colitis 13, 510-524 (2018).

3. De Souza, H. S. P. et al. The IBD interactome: an integrated view of aetiology, pathogenesis and therapy. Nat. Rev. Gastroenterol. Hepatol. 14, 739-749 (2017).

4. Sartor, R. B. \& Wu, G. D. Roles for intestinal bacteria, viruses, and fungi in pathogenesis of inflammatory bowel diseases and therapeutic approaches. Gastroenterology 152, 327-339 (2017).

5. Schwerd, T. et al. NOX1 loss-of-function genetic variants in patients with inflammatory bowel disease. Mucosal Immunol. 11, 562-574 (2018).

6. Geremia, A. \& Arancibia-Carcamo, C. V. Innate lymphoid cells in intestinal inflammation. Front. Immunol. 8, 1296 (2017).

7. Citalan-Madrid, A. F. et al. Cortactin deficiency causes increased RhoA/ROCK1dependent actomyosin contractility, intestinal epithelial barrier dysfunction, and disproportionately severe DSS-induced colitis. Mucosal Immunol. 10, 1237-1247 (2017).

8. Zhou, R. et al. JNK pathway-associated phosphatase/DUSP22 suppresses CD4(+) T-cell activation and Th1/Th17-cell differentiation and negatively correlates with clinical activity in inflammatory bowel disease. Front. Immunol. 8, 781 (2017).

9. Shi, T. et al. The signaling axis of microRNA-31/interleukin- 25 regulates Th1/Th17mediated inflammation response in colitis. Mucosal Immunol. 10, 983-995 (2017).

10. Sanctuary, M. R. et al. miR-106a deficiency attenuates inflammation in murine IBD models. Mucosal Immunol. 12, 200-211 (2019).

11. Kaser, A. et al. Inflammatory bowel disease. Annu. Rev. Immunol. 28, 573-621 (2010).

12. Morgan, M. E. et al. Toll-like receptor 6 stimulation promotes T-helper 1 and 17 responses in gastrointestinal-associated lymphoid tissue and modulates murine experimental colitis. Mucosal Immunol. 7, 1266-1277 (2014).

13. $\mathrm{He}, \mathrm{C}$. et al. MicroRNA $301 \mathrm{~A}$ promotes intestinal inflammation and colitisassociated cancer development by inhibiting BTG1. Gastroenterology 152, 1434-1448 (2017). 
14. Wu, W. et al. miR-10a inhibits dendritic cell activation and Th1/Th17 cell immune responses in IBD. Gut 64, 1755-1764 (2015).

15. Li, M. et al. Upregulation of miR-665 promotes apoptosis and colitis in inflammatory bowel disease by repressing the endoplasmic reticulum stress components XBP1 and ORMDL3. Cell Death Dis. 8, e2699 (2017).

16. Koukos, G. et al. MicroRNA-124 regulates STAT3 expression and is downregulated in colon tissues of pediatric patients with ulcerative colitis. Gastroenterology 145, 842-852 (2013).

17. Koukos, G. et al. A microRNA signature in pediatric ulcerative colitis: deregulation of the miR-4284/CXCL5 pathway in the intestinal epithelium. Inflamm. Bowel Dis. 21, 996-1005 (2015)

18. Pei, X. F. et al. Role of miR-22 in intestinal mucosa tissues and peripheral blood CD4+ T cells of inflammatory bowel disease. Pathol. Res. Pract. 214, 1095-1104 (2018).

19. Li, J. et al. Critical role of alternative M2 skewing in miR-155 deletion-mediated protection of colitis. Front. Immunol. 9, 904 (2018).

20. Singh, U. P. et al. miR-155 deficiency protects mice from experimental colitis by reducing T helper type 1/type 17 responses. Immunology 143, 478-489 (2014).

21. Bruinsma, I. B. et al. Regulator of oligodendrocyte maturation, miR-219, a potential biomarker for MS. J. Neuroinflamm. 14, 235 (2017).

22. $\mathrm{Hu}, \mathrm{X} . \mathrm{M}$. et al. Downregulation of miR-219 enhances brain-derived neurotrophic factor production in mouse dorsal root ganglia to mediate morphine analgesic tolerance by upregulating CaMKIlgamma. Mol. Pain 12, pii: 1744806916666283 (2016).

23. Rius, B. et al. Resolvin D1 primes the resolution process initiated by calorie restriction in obesity-induced steatohepatitis. FASEB J. 28, 836-848 (2014).

24. Recchiuti, $A$. et al. MicroRNAs in resolution of acute inflammation: identification of novel resolvin D1-miRNA circuits. FASEB J. 25, 544-560 (2011).

25. Huang, N. et al. MiR-219-5p inhibits hepatocellular carcinoma cell proliferation by targeting glypican-3. FEBS Lett. 586, 884-891 (2012).

26. Huang, L. X. et al. microRNA-219-5p inhibits epithelial-mesenchymal transition and metastasis of colorectal cancer by targeting lymphoid enhancer-binding factor 1. Cancer Sci. 108, 1985-1995 (2017).

27. Wei, C. et al. MicroRNA-219-5p inhibits the proliferation, migration, and invasion of epithelial ovarian cancer cells by targeting the Twist/Wnt/beta-catenin signaling pathway. Gene 637, 25-32 (2017).

28. $\mathrm{He}, \mathrm{C}$. et al. miR-301a promotes intestinal mucosal inflammation through induction of IL-17A and TNF-alpha in IBD. Gut 65, 1938-1950 (2016).

29. Ichiyama, K. et al. The microRNA-183-96-182 cluster promotes T helper 17 cell pathogenicity by negatively regulating transcription factor Foxo1 expression. Immunity 44, 1284-1298 (2016).

30. Deng, L. et al. Involvement of microRNA-23b in TNF-alpha-reduced BMSC osteogenic differentiation via targeting runx2. J. Bone Miner. Metab. 36, 648-660 (2018).

31. Xie, M. et al. NF-kappaB-driven miR-34a impairs Treg/Th17 balance via targeting Foxp3. J. Autoimmun. 102, 96-113 (2019).

32. Lin, H. Y. et al. STAT3 upregulates miR-92a to inhibit RECK expression and to promote invasiveness of lung cancer cells. Br. J. Cancer 109, 731-738 (2013).

33. Cheng, $X$. et al. ETS variant 5 promotes colorectal cancer angiogenesis by targeting platelet-derived growth factor BB. Int. J. Cancer 145, 179-191 (2019).
34. Pham, D. et al. The transcription factor Etv 5 controls $\mathrm{TH} 17$ cell development and allergic airway inflammation. J. Allergy Clin. Immunol. 134, 204-214 (2014).

35. $\mathrm{Yu}$, S. et al. IL-12 induced the generation of IL-21- and IFN-gamma-co-expressing poly-functional CD4+ T cells from human naive CD4+ T cells. Cell Cycle 14, 3362-3372 (2015)

36. Kubo, S. et al. Janus kinase inhibitor baricitinib modulates human innate and adaptive immune system. Front. Immunol. 9, 1510 (2018).

37. Delgoffe, G. M. et al. Interpreting mixed signals: the cell's cytokine conundrum. Curr. Opin. Immunol. 23, 632-638 (2011).

38. Nagashima, $\mathrm{H}$. et al. Regulation of interleukin- 6 receptor signaling by TNF receptor-associated factor 2 and 5 during differentiation of inflammatory CD4(+) T cells. Front. Immunol. 9, 1986 (2018).

39. Wirtz, S. et al. Chemically induced mouse models of acute and chronic intestinal inflammation. Nat. Protoc. 12, 1295-1309 (2017).

40. Zhou, G. et al. Tripartite motif-containing (TRIM) 21 negatively regulates intestinal mucosal inflammation through inhibiting $\mathrm{TH} 1 / \mathrm{TH} 17$ cell differentiation in patients with inflammatory bowel diseases. J. Allergy Clin. Immunol. 142, 1218-1228 (2018)

41. Yang, W. et al. Critical role of ROCK2 activity in facilitating mucosal CD4(+) T cell activation in inflammatory bowel disease. J. Autoimmun. 89, 125-138 (2018).

42. Huang, Z. et al. miR-141 Regulates colonic leukocytic trafficking by targeting CXCL12beta during murine colitis and human Crohn's disease. Gut 63, 1247-1257 (2014)

43. Cheng, X. et al. miR-19b downregulates intestinal SOCS3 to reduce intestinal inflammation in Crohn's disease. Sci. Rep. 5, 10397 (2015).

44. Yu, M. et al. MicroRNA-590-5p Inhibits Intestinal Inflammation by Targeting YAP. J. Crohn's. Colitis 12, 993-1004 (2018).

45. Fitzpatrick, L. R. Novel pharmacological approaches for inflammatory bowel disease: targeting key intracellular pathways and the IL-23/IL-17 axis. Int. J. Inflamm. 2012, 389404 (2012).

46. Radi, Z. A. et al. Pharmacologic evaluation of sulfasalazine, FTY720, and anti-IL-12/ 23 p40 in a TNBS-induced Crohn's disease model. Dig. Dis. Sci. 56, 2283-2291 (2011).

47. Van Der Heijden, T. et al. The IL-12 cytokine family in cardiovascular diseases. Cytokine 122, 154188 (2017).

48. Wei, B. \& Pei, G. microRNAs: critical regulators in Th17 cells and players in diseases. Cell. Mol. Immunol. 7, 175-181 (2010).

49. Liu, Y. et al. MiR-155 inhibition ameliorates 2, 4, 6-Trinitrobenzenesulfonic acid (TNBS)-induced experimental colitis in rat via influencing the differentiation of Th17 cells by Jarid2. Int. Immunopharmacol. 64, 401-410 (2018).

50. Wang, $H$. et al. miR-219 cooperates with miR-338 in myelination and promotes myelin repair in the CNS. Dev. Cell 40, 566-582 (2017).

51. Thieu, V. T. et al. Signal transducer and activator of transcription 4 is required for the transcription factor T-bet to promote $\mathrm{T}$ helper 1 cell-fate determination. Immunity 29, 679-690 (2008).

52. Ouyang, W. et al. The Ets transcription factor ERM is Th1-specific and induced by IL-12 through a Stat4-dependent pathway. Proc. Natl. Acad. Sci. USA 96, 3888-3893 (1999).

53. Wirtz, S. et al. Interleukin-35 mediates mucosal immune responses that protect against T-cell-dependent colitis. Gastroenterology 141, 1875-1886 (2011). 